\title{
The Surgical Management of Uveal Effusion Syndrome
}

\author{
A. G. CASSWELL, Z. J. GREGOR and A. C. BIRD \\ London
}

\begin{abstract}
Summary
Vortex vein decompression and/or partial thickness sclerectomies were performed on 6 eyes of 4 patients with uveal effusion syndrome. Vortex vein decompression was undertaken in 3 eyes and was followed by retinal re-attachment in two. Multiple sclerectomies were undertaken in 4 eyes of 3 patients; an initial reduction of sub-retinal fluid occurred in all eyes and retinal re-attachment followed in two. There were no complications following the procedure. The response to surgery in these eyes suggests that the approach is justified and provides further evidence that a scleral abnormality is the underlying cause of uveal effusion syndrome.
\end{abstract}

Idiopathic Uveal Effusion is a rare condition characterised by exudative detachment of the retina and choroid. ${ }^{1,2}$ The condition typically affects healthy middle aged males and is commonly bilateral. The serous retinal detachment is usually dependent on shifting subretinal fluid and there are no retinal holes. The associated choroidal detachment is annular in configuration. Striking changes may be evident at the level of the pigment epithelium with so called 'leopard spot' hyperpigmentation. ${ }^{2}$ There is no evidence of intraocular inflammation apart from vitreous cells which may occasionally be present. ${ }^{2}$ There are few anterior segment signs though episcleral vein dilatation has been reported. ${ }^{3}$

The natural history of the condition is variable. The condition follows a relapsing course and eyes with mild forms of the disease usually resolve. The prognosis is worse for patients with bullous retinal detachment and vision may not recover after resolution of the retinal detachment. ${ }^{1,5}$
Treatment of Uveal Effusion Syndrome has been disappointing in the past. Medical treatment including systemic steroids and antimetabolites have been ineffective $e^{2,5}$ and surgical treatment appropriate to rhegmatogenous retinal detachment can be disastrous and may lead to loss of vision. ${ }^{1,2,7,9,10}$

There is increasing evidence that abnormal sclera is responsible for Uveal Effusion Syndrome and recently scleral surgery involving vortex vein decompression and sclerectomies has appeared to be effective in a few patients. ${ }^{3,6}$

The purpose of this paper is to report our experiences with scleral surgery in a series of patients with Uveal Effusion Syndrome.

\section{Patients and Methods}

The patients had Uveal Effusion Syndrome and had undergone scleral surgery. No patient had evidence of the following: posterior scleritis, orbital inflammatory disease, arterio-venous fistula, recent panretinal photocoagulation, surgery or 
trauma, a drug reaction to sulphonamides or acetazolamide.

\section{Case 1}

A 48 year old man suffered recurrent self limiting retinal detachments in both eyes for 14 years. No associated systemic abnormality was found on investigation and the detachments were initially thought to be either due to central serous retinopathy or a form of Harada's Syndrome. He developed persistent bilateral retinal detachments associated with choroidal detachments and his vision deteriorated to hand movements with the right eye and perception of light with the left. Treatment with high dose systemic steroids and plasmapheresis was ineffective. He underwent surgical exploration. Only 2 vortex veins were found in each eye and these entered the sclera close to the optic nerve heads. The vortex vein canals were de-roofed and this revealed a $20 \mathrm{~mm}$ intra-scleral course and abnormally thick sclera. Sub-retinal fluid was drained and air injected into each eye. The retina in the left eye flattened but total reattachment was prevented by sub-retinal bands. The vision, though still recorded at hand movements, was markedly improved functionally. Subsequently a vitrectomy was carried out to release the sub-retinal bands but the retina became totally detached. In the right eye, the vortex vein decompression was complicated by incision of one vortex vein which required cautery; nevertheless, the retina became re-attached over a period of several weeks, with improvement of vision to finger counting.

\section{Case 2}

A 44 year old man developed bilateral Uveal Effusion Syndrome. He was moderately myopic and his visual acuity was $6 / 36$ with the right eye and $6 / 60$ with the left. He had sub-total retinal detachments in both eyes which were more extensive in the left eye. Investigations failed to reveal an underlying cause. Initially he underwent an exploratory operation; the vortex veins were found to be extremely tortuous and it was possible to find only one scleral entry site, at the equator, in each eye. The vortex vein in the left eye was decompressed and this had no effect.

Scleral resections in all 4 quadrants of each eye were performed. The technique used was to fashion a 5 by $7 \mathrm{~mm}$ scleral flap at the equator in each quadrant (Fig. 1). The entry sites of the vortex veins and their presumed intrascleral course were specifically avoided. The scleral flaps were dissected to an almost full thickness depth, so that the blue colour of the choroid was clearly visible. Full thickness sclerostomies were not performed. As the sclera was resected, the deep scleral bed became wet with fluid passing out of the choroid.

The retinae became reattached after 1 month in the right eye and 4 months in the left with visual acuity improving to $6 / 12$ and $6 / 36$ respectively and the retinae remained re-attached.

Case 3

A 45 year old woman with long-standing systemic lupus erythematosis presented with a one year history of deteriorating vision. The right eye was blind (no perception of light) and vision with the left eye was reduced to hand movements. She had a sub-total retinal detachment with marked shifting of the subretinal fluid in the left eye. Ultrasonography demonstrated abnormally thick posterior coats of the eye (Fig. 2a).

At surgery the vortex veins were found to be abnormally dilated and tortuous but normal in number and distribution. Sclerectomies were performed in each quadrant and this demonstrated abnormally thick sclera. Immediately post-operatively, a reduction of sub-retinal fluid was evident and the vision improved to $2 / 36$. Over the following 4 weeks the sub-retinal fluid re-accumulated and the vision deteriorated to the pre-operative level. The eye was re-explored one month following surgery and a layer of fibrous tissue was found overlying each of the sclerectomies. The fibrous tissue was excised and the sclera was thinned down at the original sites. Post operatively there was once again a transient visual improvement and reduction in sub-retinal fluid for a few weeks before returning to the preoperative state.

\section{Case 4}

An 11 year-old boy developed a left convergent squint at one year of age and a retinoschisis was diagnosed. When seen at Moorfields aged 11 years, the vision in the left eye was reduced to finger counting. There was a sub-total retinal detachment with marked sub-retinal exudation at the posterior pole and a small area of epiretinal membrane in the inferotemporal quadrant. The eyes were quiet and there was no evidence of pars planitis, schisis or telangiectasis. Ultrasound measurement of the axial length was $17.3 \mathrm{~mm}$ and gross thickening of the posterior coats of the eye was evident (Fig. 2b).

At operation abnormally thick sclera was noted and 4 sclerectomies were fashioned. Postoperatively the sub-retinal fluid diminished over the first 4 weeks and the visual acuity improved to. $6 / 36$.

\section{Discussion}

We have operated on 6 eyes of 4 patients with 


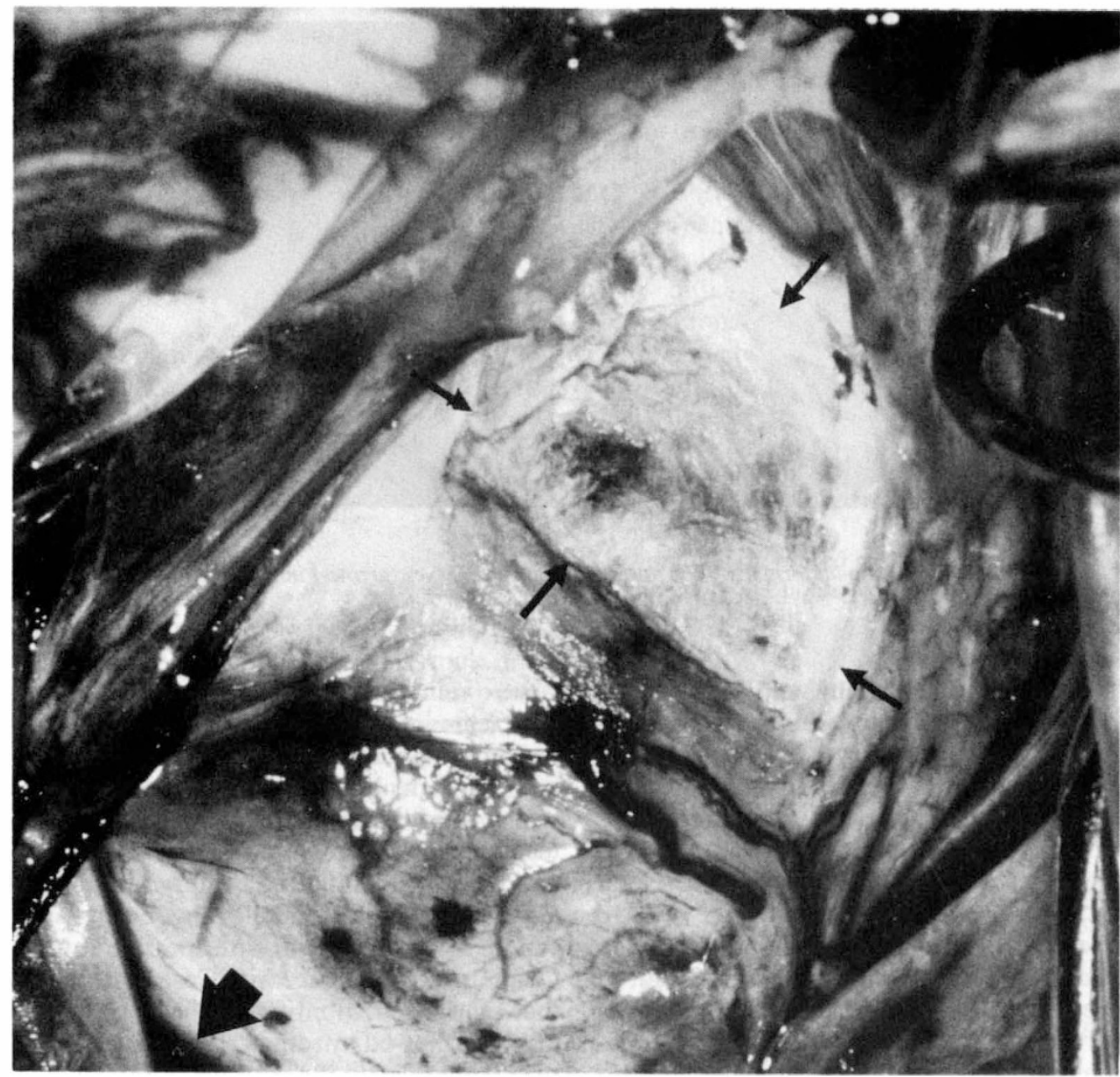

Fig. 1. Photograph of a partial thickness scleral resection (small arrows) at the equator (large arrow denotes the limbus).

Uveal Effusion Syndrome. Abnormalities of the vortex veins and abnormally thick sclera were common to all eyes. Vortex vein decompression was successful causing retinal re-attachment in 2 eyes but was technically difficult to perform. Following scleral resection, the retina became re-attached in 2 eyes though all 4 showed initial reduction in subretinal fluid and a visual improvement.

Theories as to the pathogenesis of Uveal Effusion Syndrome derive from the observation that Uveal Effusion Syndrome is more common in nanophthalmic eyes ${ }^{7}$ in which the sclera is abnormally thick. ${ }^{7,8}$ It was considered that the abnormally thick sclera compressed the vortex veins and impeded drainage. This concept is supported by a recent case report of Uveal Effusion in Hunters Syndrome (Type II mucopolysaccaridosis) ${ }^{6}$ in which the scleral thickness is increased by infiltrating mucopolysaccharides.

Brockhurst carried out vortex vein decompression in nanophthalmic eyes and this was reported to be followed by retinal flattening. ${ }^{9}$ Gass attempted vortex vein decompression in eyes that were not nanophthalmic and found that thick sclera was present in these eyes too. ${ }^{2}$ Gass, however, found vortex vein decompression to be a difficult surgical procedure but found that the 


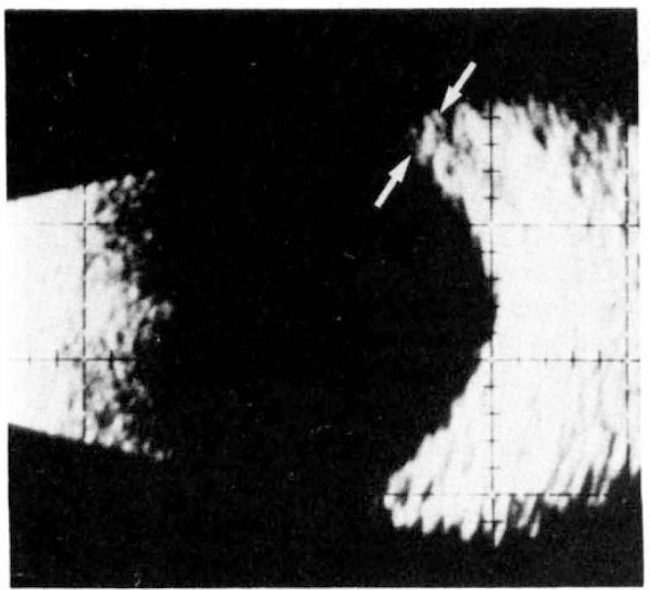

a

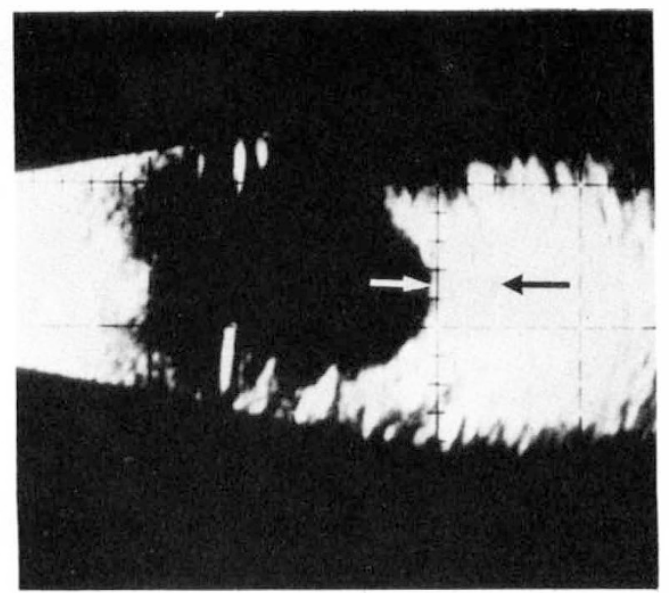

b

Fig. 2. Ultrasound showing abnormally thick posterior coats of the eyes (arrows) in Case 3 (a) and Case 4 (b).

effusion settled following surgery even in cases in which the vortex veins had been transected. He concluded it was the full thickness scleral incisions rather than any change in venous outflow that caused retinal re-attachment. This led to the hypothesis that the primary cause of uveal effusion is a scleral abnormality which predisposes the eye to vortex vein obstruction and acts as a barrier to the diffusion of extra vascular protein out of the eye. He devised the alternative therapy of scleral thinning. ${ }^{3}$ This took the form of 4 equatorial partial thickness scleral flaps removed from each quadrant without disturbing vortex veins. In addition a central sclerostomy was made. In the patient Gass reported, the retinae re-attached in both eyes with recovery of vision. ${ }^{3}$ Recently a case of Hunters Syndrome with uveal effusion had similar surgery which was followed by re-attachment of the retina. ${ }^{6}$ Brockhurst had not found that sclera was abnormal in eyes that were not nanophthalmic and had not found that scleral surgery had been effective in one case described. ${ }^{3}$

Our surgical experience has been similar to that of Gass. Prior to surgery on Case 1, it was generally felt that patients with Uveal Effusion Syndrome respond badly to surgery with the possible exception of patients with nanophthalmos. It was encouraging that vortex vein decompression was followed by reattachment of both retinae but the lack of major visual improvement was disappointing and may have been related to the long duration of the detachment. However, the surgical technique is difficult and in Case 2, one vein was incised and required cautery. This operation was ineffective. Following Gass' publication, ${ }^{3}$ scleral resections were carried out in Case 2. Our experience with Case 2 suggested that scleral resection is technically easy and may be followed by retinal re-attachment and recovery of vision if performed early enough after development of the detachment.

There was a clear though transient beneficial response to surgery in Case 3. Possibly a more successful and long-lasting effect might have been achieved if larger or thicker sclerectomies had been performed, if full thickness sclerostomies had been performed or if the inflammatory response had been suppressed by steroids. Brockhurst used high doses of systemic steroids for the first week post-operatively. ${ }^{9}$ Gass described the use of a punch to make a sclerostomy, ${ }^{3}$ but we did not make full thickness scleral incisions.

Surgery in Case 4 was followed by an improvement in vision with some reduction of sub-retinal fluid but this retina had been detached for several years and there was coincident epiretinal membrane. 
Table I Differential diagnosis of patients with serous retinal detachment

(1) Central serous retinal detachment with bullous retinal detachment

(2) Harada's disease

(3) Diffuse melanoma or carcinoma of the uveal tract

(4) Reactive lymphocytic hyperplasia of the uvea

(5) Rhegmatogenous retinal detachment with uveal detachment

While we cannot rule out the possibility that spontaneous recovery occurred in the patients reported here, the temporal relationship between surgery and resolution suggests that surgery brings about reattachment of the retina in a proportion of patients.

\section{Management}

When faced with the management of this rare and difficult condition, the diagnosis must first be established. Although diabetes and collagen disorders have been implicated as underlying causes, ${ }^{1}$ no consistent systemic conditions have been reported. In the absence of a clear cause such as trauma, surgery or hypotony, there are conditions that simulate this condition (Table I) that have to be excluded. ${ }^{3}$ Most of these conditions have associated clinical signs to indicate that primary diagnosis and fluorescein angiography can be helpful. As the natural history is relatively benign, patients should be observed if their vision remains good and their macula remains attached. If the vision is deteriorating steadily, we recommend ultrasonography to identify abnormal thickness of the posterior coats of the eye and to identify the short axial length of nanophthalmic eyes. At exploratory surgery, any abnormalities of the vortex veins can be identified and the thickness of the sclera can be assessed by cutting down onto the choroid. If the sclera is found to be thick (greater than $2 \mathrm{~mm}$ ), we recommend the fashioning of partial thickness sclerectomies in each quadrant, avoiding the vortex veins. There appears to be enough evidence to support this surgical approach. It is neither technically difficult nor yet results in serious complications, in contrast to the traditional view of detachment surgery. Clearly it would be desirable to perform a controlled trial of this treatment especially in view of the variable natural history, but it is unlikely that this will be possible in view of the rare nature of the disorder.

This work was supported in part by the Friends of Moorfields; AGC holds a Friends of Moorfields Research Fellowship.

\section{References}

${ }^{1}$ Schepens CL and Brockhurst RJ: Uveal effusion. I. Clinical picture. Arch. Ophthalmol. 1963, 70, 189-210.

${ }^{2}$ Gass JDM and Sulayman J: Idiopathic serous detachment of the choroid, ciliary body and retina (uveal effusion syndrome). Ophthalmology 1982, 89, 1019-31.

${ }^{3}$ Gass JDM: Uveal effusion syndrome: a new hypothesis concerning pathogenesis and technique of surgical treatment. Trans. Am. Ophthalmol. Soc. 1983, 81: 247-60.

${ }^{5}$ Davies EWG: Annular serous choroidal detachment. Mod. Probl. Ophthal. 1979, 20: 2-5.

${ }^{6}$ Vine AV: Uveal effusion in Hunter's syndrome. Retina 1986, 6: 57-60.

${ }^{7}$ Brockhurst RJ: Nanophthalmos with uveal effusion. Arch Ophthalmol. 1975, 93: 1289-99.

${ }^{8}$ Calhoun FP: The management of glaucoma in nanophthalmos. Trans. Am. Ophthalmol. Soc. 1975, 73: 98-122.

${ }^{9}$ Brockhurst RJ: Vortex vein decompression for nanophthalmic uveal effusion. Arch. Ophthalmol. 1980, 98: 1987-90. 